\title{
Precision Ellipsometry for Quantitative Real-Time Monitoring of Molecular Layers
}

\author{
Nikolai L Yakovlev* \\ Institute of Materials Research and Engineering, Agency for Science, Technology and Research, Singapore
}

*Corresponding author: Nikolai L Yakovlev, Institute of Materials Research and Engineering, Agency for Science, Technology and Research, 2 Fusion polis Way, Innovis, \#08-03, 138634, Singapore.

Received Date: July 26, 2019

Published Date: August 02, 2019

\begin{abstract}
Precision ellipsometry (PREL) is optical technique that uses polarization modulator to measure changes in the state of light polarization reflected off a surface of a material. Based on new modulation concept, a polarization modulator was made, which is compact, non-magnetic, consuming low power. With this modulator, a portable PREL system was made and was shown to have sensitivity at molecular level via experiments on attachment of various molecules on silicon surface from air and from water. Real-time measurements of kinetics of molecular binding gave insights into mechanisms of the binding and gave directions to improvement of technology of fabrication of respective materials.
\end{abstract}

\section{Introduction}

Ellipsometry is an analytical technique that measures polarization of light reflected off the surface of a material. In contrast to optical techniques that measure light intensity, ellipsometry measures phase shift between components of polarization of reflected light [1], this provides high sensitivity and possibility to measure parameters of thin films on reflecting surfaces. It has been successfully applied to huge variety of films and substrates; particularly molecular layers were investigated on inorganic substrates [2,3], organic substrates [4,5] and even biological objects [6].

Even higher sensitivity has been achieved using modulation of polarization; this technique is called precision ellipsometry (PREL). Here the polarized light beam passes through a polarization modulator, which creates AC component in the intensity of the light; the AC amplitude is proportional to either ellipticity or rotation of the polarization, and it is extracted by phase- lock (or lock-in) amplifier. Traditionally PREL was realized using photoelastic modulator [7]. Since then, the progress in lasers and photodetectors made them significantly smaller in size. Recently a compact polarization modulator has been designed based on new modulation concept; it is completely non-magnetic and was first used for magneto-optical experiments [8].

Thus, with semiconductor laser and compact polarization modulator, PREL systems became simpler, cheaper and even portable, while providing sensitivity in sub-nanometer range suitable for monitoring of molecular layers. Here we present the results of PREL measurements of adsorption of organic molecules on silicon substrates from air in section 3 and from water in section 4.

\section{Experimental}

In the PREL system, the light from a laser pointer at wavelength $\nabla=650 \mathrm{~nm}$ passes through a polarizer oriented at $45^{\circ}$ to the plane of incidence and goes to the sample at incident angle of $60^{\circ}$; the reflected light passes through a quarter wave retarder, then polarization modulator and reaches photodetector. As follows from single layer ellipsometric model [1], the phase shift $\Delta$ between sand p-components of reflected light (or ellipticity) is proportional the thickness $d$ of the layer, if $d r \ll$. Hence the retarder is placed to convert the ellipticity into rotation of polarization, which is measured by the modulator and phase-lock amplifier. And hence, the signal from the phase-lock amplifier is also proportional to the thickness of the layer. The optical and modulation system as well as calibration and measurement principles are described in detail in ref [9].

After demonstration of high sensitivity of PREL in air, we were asked by our colleagues, whether similar experiments could be done in water, since many chemical and bio-interactions occur in liquids. For experiments in water, a special fluidic system was made 
[10]. It comprises source reservoir of solvent with flow control valve, optical cuvette with glass windows and drain reservoir. The substrate was immersed into the cuvette, solvent flowed through pipes and solutions were injected into the cuvette using a pipette.

Silicon substrates were washed $\mathrm{H} 2 \mathrm{O}+\mathrm{H} 2 \mathrm{O} 2+\mathrm{NH} 4 \mathrm{OH}=2+1+$ 1 solution, which creates a thin oxide layer on the surface [11]. They were kept in deionized water before experiment in water. Before experiments in air, the substrates were cleaned in oxygen plasma and installed in PREL within 10 minutes.

Quantitative data of adsorbed layers were obtained converting the recorded signal into layer thickness using standard ellipsometric formulae [1], taking refractive indices of silicon 3.85, that of water 1.33 and that of organic molecules between 1.42 and 1.44. Thus, vertical scale in the figures shows the thickness of the layers. Note also, if molecules do not cover the surface completely, the fractional coverage appears in PREL as fractional thickness.

\section{PREL in Air}

To examine the sensitivity of our PREL system, we performed experiments with the substrate in laboratory atmosphere and blowing tetra-fluoro-ethane gas from a canister of a gas duster (Model 1671-10S, Brand Tech Spray), which is commonly used to remove dust or to dry a surface. The duration of blowing was several seconds from the distance of several centimeters. During the blowing a peak of polarization signal was observed most likely due to mechanical pressure of the gas flow on the substrate. However, after stopping the blow, an increment of thickness with respect to preceding level was recorded, Figure 1. The longer was blowing duration, the higher was the increment. This shows that molecules were permanently attached to the surface (Figure 1).

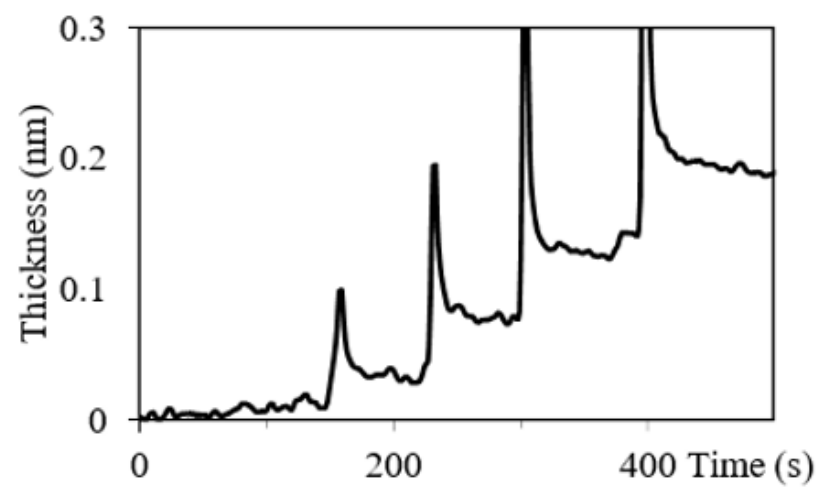

Figure 1: Adsorption of tetra-fluoro-ethane on oxidised silicon surface in air; peaks correspond to sequential blowing of gas duster for the durations of $1,2,3$ and 5 seconds.

To do molecular adsorption experiments in more reproducible conditions, we took advantage of small size of the system and placed it in an acrylic box. At certain time, marked "in" in Figure 2, a Petri dish with volatile liquid was placed into the box, the box was closed and increment of thickness was observed. At certain time marked "out", the box was opened, the dish was removed and decrease of thickness was observed (Figure 2).

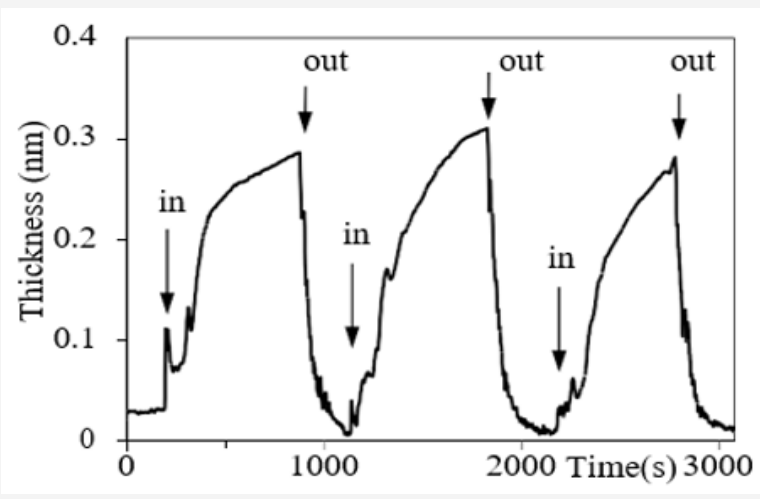

Figure 2: Adsorption and desorption of propanol on oxidized silicon surface in air.

Experiments with propanol, ethanol and methanol showed two processes in the adsorption. One process is fast, in the range of dozens of seconds, defined by diffusion of vapor to fill the box; the other is slow, in the range of hundreds of seconds, defined by substitution of previously adsorbed molecules by those from the vapor [9].

The experiments in air, Figures 1 and 2, show that the noise level is far below $0.01 \mathrm{~nm}$ and sensitivity of the system is enough to measure fractions of molecular layers. Thus, with appropriate functionalizing of the substrate, PREL has considerable potential in detection or monitoring of volatile compounds.

\section{PREL in Water}

Starting PREL experiments in water, we first took amino-propylsilane (APS), which is commonly used to functionalize glass surface with amino-groups that can bind a variety of subsequent molecules. Solution of APS was made by dissolving 3-amino-propyl-trimetoxysilane in deionized water and it was used at concentration $5 \mathrm{mMol}$. Another molecule also used as anchor layer is poly-ethylene-imine (PEI) that has branched structure with numerous flexible aminogroups. It was used at the concentration of $10 \mathrm{mMol}$ (counted per monomer unit, which is $0.43 \mathrm{mg} / \mathrm{mL}$ ).

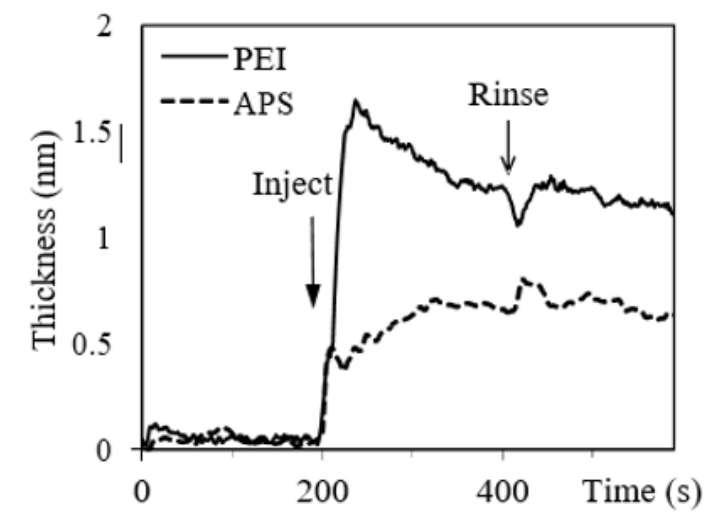

Figure 3: Adsorption of amino-propyl-silane (APS) and polyethylene-imine (PEI) on oxidized silicon in water.

Experiments shown in Figure 3 were carried out as follows: water was flowing through the cuvette during $200 \mathrm{~s}$; then the flow 
was stopped and the solution of either APS or PEI was injected. After the next $200 \mathrm{~s}$, the flow was resumed, and the solution was rinsed away.

It is seen that APS is adsorbed monotonously with time and reached the final thickness of $0.7 \mathrm{~nm}$. As a largermolecule, PEI reaches larger final thickness of $1.2 \mathrm{~nm}$; however, during the attachment, it gives a peak and then decreases. This non-monotonous kinetics can be explained takin into account that PEI forms multiple bonds to the substrate and these are reversible hydrogen bonds. Thus, at relatively high concentration, the surface is quickly covered with molecules at different orientations. Subsequently those molecules, which have already more bonds, push out those, which have less bonds, and the average thickness decreases.

Real-time measurement of kinetics of processes on the surface is useful to determine optimal process time, concentrations and other parameters. In case of PEI, the data in Figure 3 gave the direction to decrease the concentration and, really, lower concentration gave monotonous attachment seen in Figure 4.
Such advantage of PREL as real time monitoring of depositions and attachments gives considerable help in studies of multilayer formation. One of numerous applications of the multilayers is fabrication of microcapsules, which are made of alternating polyelectrolyte layers [12], or of a pair of molecules that by some means attach to each other [13]. Here we present two examples of the formation of multilayers.

The first is poly-electrolyte multilayer from solutions of polycation poly-allylamine-hydrochloride (PAH) at the concentration $0.5 \mathrm{mMol}$ (counting per monomer unit) and poly-anion polystyrene-sulfonate (PSS) at $0.5 \mathrm{mMol}$ (Figure 4). These molecules bind to each other by 3 hydrogen bonds between sulfonic group and protonated amino group, thus the attachment was done in acidic conditions at $\mathrm{pH} 5$. It is seen that adding of each layer gives an increment of total thickness: around $0.7 \mathrm{~nm}$ from $\mathrm{PAH}$ and 1.7 $\mathrm{nm}$ from PSS; the attachment is monotonous, and the increment is irreversible (Figure 5).

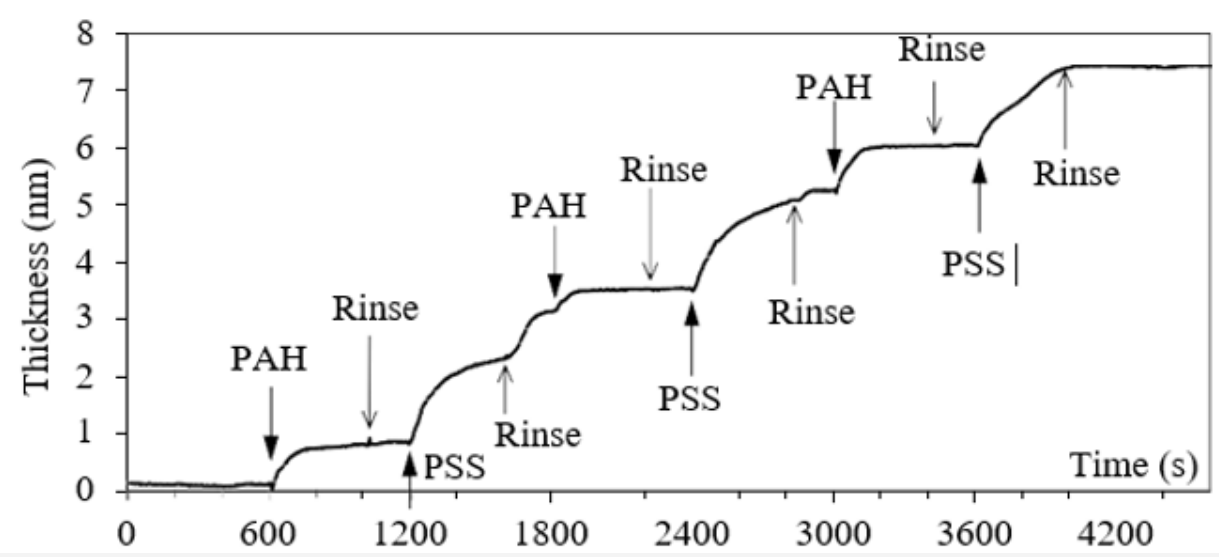

Figure 4: Deposition of poly-electrolyte multilayer of poly-allylamine-hydrochloride (PAH) with poly styrene-sulfonate (PSS) on oxidized silicon substrate in water; filled arrows show injections, open arrows show rinsing.

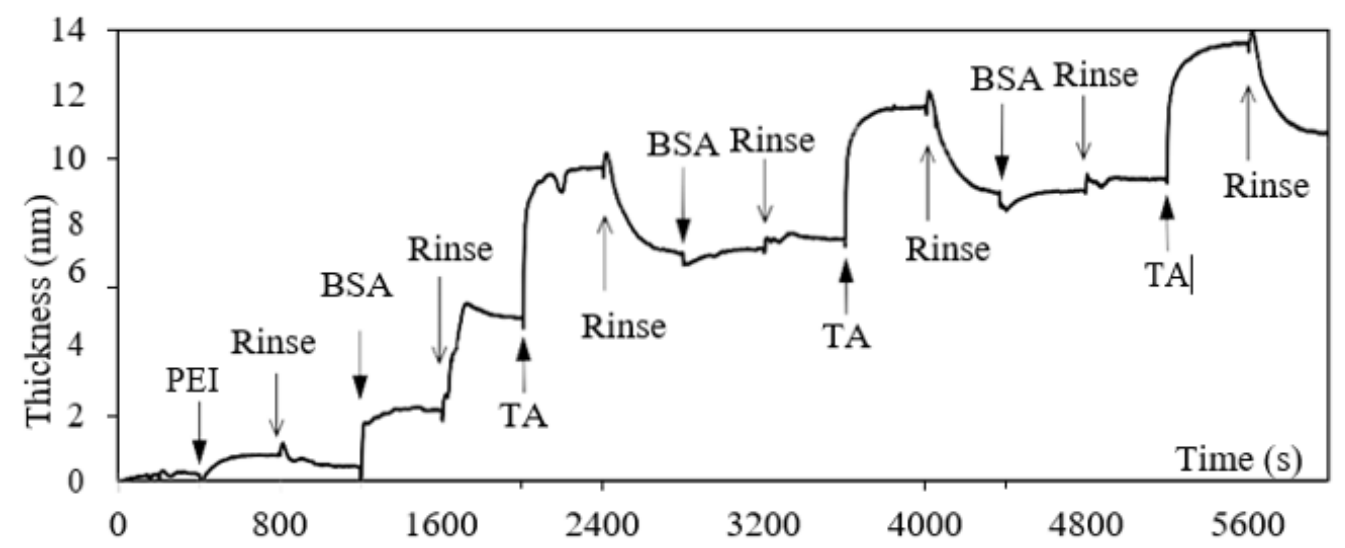

Figure 5: Deposition of poly-ethylene-imine (PEI) anchor layer and bovine serum albumin (BSA) with tannic acid (TA) multilayer on oxidized silicon substrate in water; filled arrows show injections, open arrows show rinsing

The second example is the multilayer from solutions of bovine serum albumin (BSA) at $4 \mathrm{mg} / \mathrm{ml}$ and tannic acid (TA) at $0.6 \mathrm{mg} /$ $\mathrm{ml}(0.35 \mathrm{mMol})$ on the anchor layer of PEI, Figure 5. The evident feature of this record is periodic dependence of TA adsorption and desorption. From 4nm of TA adsorbed on BSA, 1.5nm remains after rinsing in every period. Adsorption of BSA on TA is only $0.4 \mathrm{~nm}$, while the first BSA layer on PEI is $4 \mathrm{~nm}$ thick; that corresponds to the size of BSA molecule and means a complete layer. Apparent 
increase of thickness during rinsing of the first BSA layer at 1600 $\mathrm{s}$ is not clear yet, though it always comes out like that. Hence for quantitative consideration, thickness values written above are taken at the end of each rinsing.

Possibility of monitoring of several molecules in one run opens the way for real-time measurement of chemical reactions and molecular interactions. For that, one of the molecules can be immobilized on a reflecting surface and the other supplied in solution. From measurement of kinetics of attachment or detachment, it is possible to investigate the interactions at various environmental conditions, concentrations, $\mathrm{pH}$, salinity, temperature, etc., etc.

\section{Conclusions}

Based on new concept of polarization modulation, a compact modulator was created, which enabled making PREL system simple, cheap and portable. It was shown to have sensitivity at sub-nanometer and molecular level and capability of real-time measurement of kinetics of molecular interactions.

Experiments on molecular adsorption from air on silicon substrates revealed processes of substitutional adsorption. They showed that this PREL is sensitive even to fractions of molecular layers and has potential in detection or monitoring of volatile compounds.

Experiments in water also showed sensitivity of PREL at molecular level. Measurements of kinetics of molecular binding gave insights into mechanisms of the binding. Real-time measurements of molecular layer formation give directions to improvement of technology of fabrication of respective materials.

\section{Acknowledgements}

The authors are grateful to the students Su Jingyi and Kristin Kwok for pioneering experiments with PREL system, John Kwok, Huo Chin Quek and Tong Hien Chi for experiments in air, Seah Ying Hang, Wong Han Xuan, Chang Jia Geng, Jessalyn Low and Yun Waddy Soe for experiments in water. Special thanks are expressed to Grace Cheong for assembling the portable PREL setup. Help of IMRE colleagues: Laura Sutarlie, Lau Hooi Hong, Maxim Kiryukhin and Henry Goh is greatly appreciated. The work was supported by Agency for Science, Technology and Research, Singapore.

\section{Conflict of Interest}

No conflict of interest.

\section{References}

1. Harland G Tompkins, Eugene A Irene (2005) Handbook of Ellipsometry. Springer-Verlag GmbH, USA.

2. ML Gee, TW Healy, LR White (1989) Journal of Colloid and Interface Science 131(2): 514-516.

3. TM Byrne, S Trussler, MA McArthur, LB Lohstreter, Zhijun Bai, et al. (2009) A new simple tubular flow cell for use with variable angle spectroscopic ellipsometry: A high throughput in situ protein adsorption study. Surface Science 603(18): 2888-2895.

4. T Berlind, M Poksinski, O Tengvall, $\mathrm{H}$ Arwin (2010) Formation and cross-linking of fibrinogen layers monitored with in situ spectroscopic ellipsometry. Colloids and Surfaces B: Biointerfaces 75(2): 410-417.

5. O Svensson, $\mathrm{T}$ Arnebrant (2012) Antibody-antigen interaction on polystyrene: An in situ ellipsometric study. Journal of Colloid and Interface Science 368(1): 533-539.

6. H Arwin (2011) Application of ellipsometry techniques to biological materials. Thin Solid Films 519(9): 2589-2592.

7. SN Jasperson, SE Schnatterly (1969) An Improved Method for High Reflectivity Ellipsometry Based on a New Polarization Modulation Technique. The Review of Scientific Instruments 40(6): 761-767.

8. N Yakovlev, A Kaveev, N Sokolov, B Krichevtsov, A Huan (2006) Novel magnetic nanostructures: Epitaxial cobalt films in transparent fluoride matrix. Current Applied Physics 6(3): 575-578.

9. NL Yakovlev, HC Quek, KM Dabrowski, WR Birch (2019) Kinetics of small molecule adsorption studied using precision ellipsometry. Surface Interface Analysis 51(7): 697-702.

10. HH Lau, R Murney, NL Yakovlev, MV Novoselova, SH Lim, et al. (2017) Protein-tannic acid multilayer films: A multifunctional material for microencapsulation of food-derived bioactives. J Colloid Interface Sci 505: 332-340

11. Ishizaka A, Shiraki Y (1986) Low Temperature Surface Cleaning of Silicon and Its Application to Silicon MBE. J Electrochem Soc 133 (4): 666-671.

12. MN Antipina, MV Kiryukhin, AG Skirtach, GB Sukhorukov (2014) Micropackaging via layer-by-layer assembly: microcapsules and microchamber arrays. Int Mater Rev 59(4): 224-244.

13. MV Lomova, AI Brichkina, MV Kiryukhin, EN Vasina, AM Pavlov, et al. (2015) ACS Appl Mater Interfaces 7(22): 11732-11740. 